\title{
Put the patient in the room, always
}

\section{Liam J Donaldson}

There used to be a time when, in a meeting to discuss the safety of healthcare, someone would stand up and say indignantly "We have been discussing safety all day and no one has used the word 'patient', not even once." Everyone would smile ruefully and acknowledge the oversight. But everyone stayed within their comfort zone-no threat, no challenge to attitudes, beliefs or practice. Then came a time when, in such a gathering, a doctor, nurse or chief executive officer would relate the experience of a patient that they knew or had heard of. It would always enrich a discussion and provide insights. But everyone was still in a comfort zone. There was no challenge and little emotion. It was all too tidy. The true, authentic patient experience was the elephant in the room. No one wanted to face the painful reality that medical errors caused tragedies that robbed families of their future.

Today we are in an era when in some gatherings (and especially the most enlightened ones) the patient or their family members are in the room. They tell their own stories, relate their own experiences, recount their own emotions. This moves outside the comfort zone. There is challenge. It can be uncomfortable. It can be raw. It can be emotional, sometimes confrontational. Those who create opportunities for this direct dialogue with patients and their families do not always have the gratitude of their colleagues. They are often resented. They are seen as bringing problems, causing difficulties, making life untidy. Their role is often a lonely one.

In Vancouver, Canada, in 2006, I gave a keynote address on patient safety, in which I showed some short video-clips of family members who had been victims of medical error talking about their experience and how it had affected their lives. I expressed my solidarity with them, especially those who had had to fight to find out the truth about the death of their loved one. After the speech, I met representatives of the Canadian Patients

Correspondence to: L J Donaldson, Richmond House, 79 Whitehall, London SW1A 2NS, UK; Liam.donaldson@ dh.gsi.gov.uk for Patient Safety Programme. ${ }^{1}$ They are people who either themselves have experienced harm from unsafe care or members of their family have. Several were mothers of children who had been killed by medical errors. As we gathered for coffee before the meeting, one mother approached me and said quietly, "I listened to your speech. Today, you have validated my experience."

Over the next few minutes, one by one, others came over and said similar things. They spoke of "walking on eggshells" when they tried to relate their family's experience to healthcare providers. They spoke of how many doors were shut to the patient. They spoke about the absence of a safe environment to tell their stories. These were brave and extraordinary people. They have had the courage to rise above their own intense feelings of grief and loss to want to work with those providing unsafe care to make it safer for someone else's children. In short, they wanted to do all they can to ensure that no other family has to experience the tragedy which happened to them. In the words of one father whose young daughter had died following a misdiagnosis of a serious illness "Patient safety is my life now."

Let us stop the defensiveness, the acceptance that serious change cannot happen. Let us stop closing doors on our patients. Despite the unique and powerful insights of such people around the world, they are disempowered and face a huge monolith of vested interest and tradition. It is not surprising that behind those who do step forwards to represent the patient's experience are many thousands of others who have been touched by tragedy and suffer in silence.

Lee Duggins was the first child to die from the maladministration intrathecally of the cytotoxic drug vincristine. He died in 1975 in Doncaster, England. The investigation into his death was unsatisfactory. His parents were denied proper access to the hearing. They had no opportunity to speak of their experience. Thirty years later when they spoke to me, their emotions were still raw from this tragedy that had scarred their lives. The healthcare system had killed their son and then deserted and shunned them as victims. A video-clip put Mrs Duggins in the room in Vancouver. In her own words:

\section{"Lee was the first tragic death to take place and hopefully Wayne Jowett will be the last..."}

Wayne Jowett died of the same error in Nottingham, England about 30 years after Lee Duggins. ${ }^{2}$

Patients have much more to offer than visceral reminders to healthcare workers, administrators and policy makers that patient safety is a topic that needs to be taken much more seriously. Patients can also have a meaningful role in improving safety. Patients and their families often have a unique perspective on their experience of healthcare and can provide information and insights that healthcare workers may not otherwise have known.

Patients must be at the heart of patient safety. This is the route that the DanaFarber Cancer Institute in Boston has taken. A high-profile patient death in 1994 as a result of an error, ${ }^{3}$ launched a profound re-engineering of how the organisation viewed, understood and managed patient safety. The active involvement of patients has been at the centre of the DanaFarber safety mission. ${ }^{4}$ Patient safety advisory councils ensure that patients and their carers are actively involved in the design, implementation and assessment of safety initiatives and care overall.

On the international stage the World Health Organization World Alliance for Patient Safety has established as one of its core activities a Patients for Patient Safety programme. ${ }^{5}$ Led by patients, this initiative aims to ensure that patients and families who have been the victims of medical error are empowered to lead, challenge and inspire as active partners in the international patient safety movement. A global network of patient champions is growing. ${ }^{6}$ These champions, drawn from more than 50 different countries, are advocating for improvements in patient safety through open dialogue and partnership with patients and their families.

We need to go further. We need to think of those who are not able to step into such leadership positions. What of the families that are left behind-the mothers, the fathers, the sons, the daughters? What should we do for them? We cannot bring their loved one back so what should we do? 
There are some things we should never do. We should not turn our backs on them. We should not reject them. We should not deny them explanations and we should never, ever be afraid to tell them the truth. Beyond this, we should ask them to be involved: to bring the wisdom of their experience to help design safer healthcare institutions and to bring about that culture change that is so badly needed. At the global level in major strategic programmes, at the national level in the determination of health policy priorities, at the hospital level in the running of the institution, at the clinical level in the design of the patient pathways, the patient should be in the room. Not just sometimes, but always.

Competing interests: None.

Qual Saf Health Care 2008;17:82-83.

doi:10.1136/qshc.2007.025262

\section{REFERENCES}

1. Canadian Patient Safety Institute. http://www. patientsafetyinstitute.ca/index.html (accessed 13 February 2008).
2. Toft B. External inquiry into the adverse incident that occurred at Queen's Medical Centre, Nottingham, 4 January 2001. London: Department of Health, 2001.

3. Hatlie MJ, Sheridan SE. The medical liability crisis of 2003: must we squander the chance to put patients first? Health Aff (Millwood) 2003; 22:37-40.

4. Dana-Farber Cancer Institute. http://www.dfci. harvard.edu/pat/patient-safety/patient-safety-journey. html (accessed 13 February 2008).

5. World Health Organization. World Alliance for Patient Safety. http://www.who.int/patientsafety (accessed 13 February 2008).

6. World Health Organization. Patients for Patient Safety. http://www.who.int/patientsafety/patients for_patient (accessed 13 February 2008).

\section{BMJ Careers online re-launches}

BMJ Careers online has re-launched to give you an even better online experience. You'll still find our online services such as jobs, courses and careers advice, but now they're even easier to navigate and quicker to find.

New features include:

- Job alerts - you tell us how often you want to hear from us with either daily or weekly alerts

- Refined keyword searching making it easier to find exactly what you want

- Contextual display - when you search for articles or courses we'll automatically display job adverts relevant to your search

- Recruiter logos linked directly to their organisation homepage - find out more about the company before you apply

- RSS feeds now even easier to set up

Visit careers.bmj.com to find out more. 